\title{
Mémoire quantique cristalline pour la lumière
}

Thierry Chanelière (thierry.chaneliere@u-psud.fr)

Laboratoire Aimé Cotton, UMR CNRS 9180, Université Paris-Sud 11, Bât. 505, 91405 Orsay Cedex

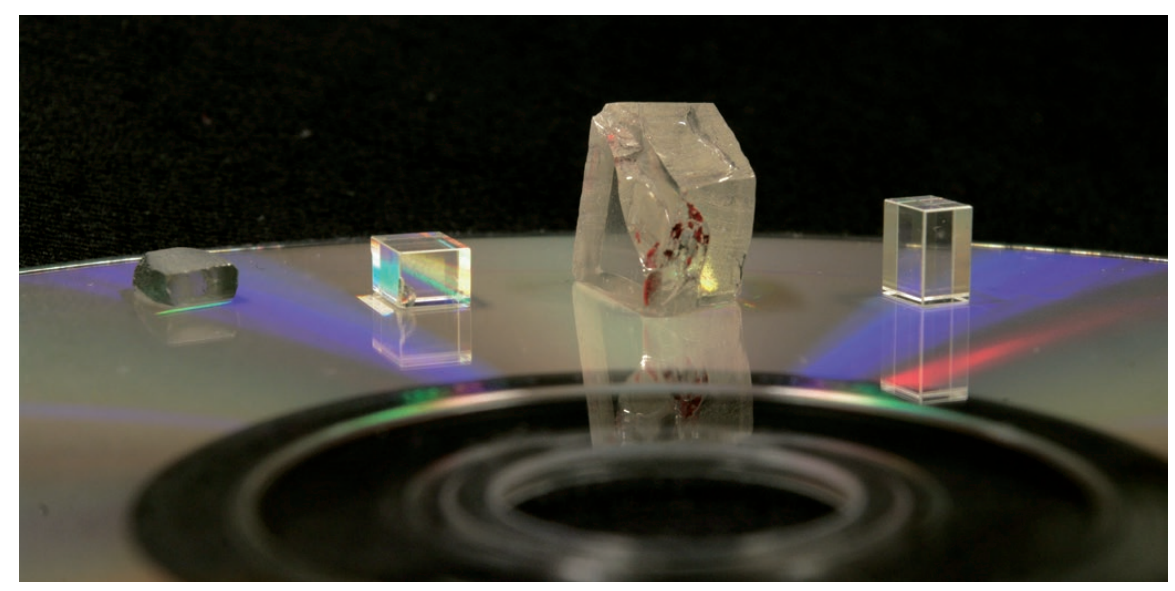

On explique dans cet article comment des cristaux

\section{faiblement dopés aux ions}

de terre rare peuvent être

\section{utilisés comme mémoire}

quantique pour la lumière.

Après avoir présenté la notion

d'information quantique

en montrant la différence

avec son analogue classique

omniprésent dans nos vies,

on explique pourquoi les ions

de terre rare insérés dans

des matrices cristallines

solides peuvent être

d'excellents porteurs

d'information.

On présente enfin un

exemple récent de réalisation

d'une telle mémoire optique,

compatible avec le réseau

actuel de télécommunication.

Cristaux synthétiques dopés aux ions $\mathrm{Tm}^{3+}$ ou $\mathrm{Er}^{3+}$ pour le stockage d'information quantique. De droite à gauche, Er:CaWO $0_{4}$, Im:Luz $\mathrm{O}_{3}$ (brut de découpe), Im:LiNb0 $0_{3}$ et $\mathrm{Tm}: \mathrm{Y}_{2} \mathrm{O}_{3}$. Ce dernier, plus petit, légèrement grisé, est de moins bonne qualité optique. Sa réalisation est une prouesse technique, puisque sa température de fusion est de $2400^{\circ} \mathrm{C}$, à comparer à $1200^{\circ} \mathrm{C}$ pour LiNb03. Le Compact Disc qui sert de présentoir est un clin d'œil à l'introduction de l'article.

\section{L'information quantique portée par la lumière}

Le développement d'internet n'aurait pas été possible sans l'utilisation de fibres optiques pour transporter l'information. Dans ces « tuyaux » circulent des impulsions lumineuses qui forment une alternance de 0 et de 1 à la base de l'encodage binaire d'information classique. Chaque impulsion contient un très grand nombre de photons. La description en termes ondulatoires classiques est alors bien adaptée. Mais que se passe-t-il si l'on réduit à 1 le nombre de photons dans une impulsion? On doit alors utiliser la mécanique quantique pour décrire la lumière. Aux états classiques 0 et 1 se substituent les fonctions d'onde $\mid 0>$ et $\mid 1>$. La vraie différence entre information classique et quantique n'est pas qu'une différence de notation. En mécanique quantique, il est possible d'écrire toutes les combinaisons linéaires $|\psi\rangle=\mathrm{a}|0>+\mathrm{b}| 1>$ entre les deux états, alors qu'une particule classique est dans l'état 0 ou 1 exclusivement. Pour cette superposition cohérente $|\psi\rangle$, on parle alors de qubit pour marquer le caractère quantique du porteur d'information. Cette définition formelle ouvre un champ d'applications, celui de l'information quantique.

L'application phare du domaine est la sécurisation des communications : la cryptographie quantique [1]. La sécurité du message est alors assurée par la théorie de la mesure en mécanique quantique : en effectuant des mesures, un espion malveillant modifierait immanquablement l'état quantique et serait donc détecté. Des appareils de cryptographie quantique existent commercialement. Ils sont malheureusement limités à une portée de quelques centaines de kilomètres à cause des pertes résiduelles dans les fibres. Ces pertes sont certes faibles, mais problématiques lorsqu'on travaille avec un seul photon. Une solution proposée pour étendre la portée des dispositifs de cryptographie est d'en connecter des segments élémentaires par des mémoires quantiques qui jouent le rôle de relais, dits "répéteurs quantiques ». On mesure ici l'importance du développement de mémoires compatibles avec le réseau de fibres optiques actuelles. 


\section{(Dé)-cohérence}

encadré 1

La cohérence et la décohérence sont des notions profondes de mécanique quantique. À partir de deux états du système notés $\mid 0>$ et $\mid 1>$ en information quantique (par analogie aux nombres binaires 0 et 1 ), on peut écrire une superposition cohérente sous la forme $|\psi>=a| 0>+b \mid 1>$, où $a$ et $b$ sont des nombres complexes avec $|a|^{2}+|b|^{2}=1$. Les facteurs $|a|$ et $|b|$ représentent les populations des états. Prenons comme exemple une superposition à poids égal des deux états, $|\mathrm{a}|=|\mathrm{b}|=1 / \sqrt{ } 2$.

La phase globale n'ayant pas d'importance, on peut choisir a réel et écrire l'état $\mid \psi>=(|0>+\exp (i \phi)| 1>) / \sqrt{ } 2$. Le terme exp(i $\phi)$ est un terme de cohérence par excellence, car il porte la différence de phase entre les deux états de base $\mid 0>$ et $\mid 1>$.

Malgré le caractère très quantique du concept de cohérence, on peut en donner une image classique. On peut voir l'excitation atomique comme la vibration d'un électron élastiquement lié au noyau. La phase de cette vibration représente la cohérence, alors que son amplitude représente la population. Qu'est-ce que la décohérence dans ce cas-là ? C'est quand on perd trace de la phase oscillante. Comment ? Prenons un exemple fictif, mais réaliste. Supposons que l'atome baigne dans un champ magnétique fluctuant aléatoirement à cause d'un environnement agité (impuretés voisines sensibles aux phonons, par exemple). Si l'atome est sensible à l'effet Zeeman, alors l'écart d'énergie qui définit la fréquence de transition (de Larmor) se déplace de $\Delta_{\mathrm{j}}$ pendant un temps $\tau_{j}$. Ces perturbations pendant les temps $\tau_{1}, \tau_{2} \ldots \tau_{j}$ vont modifier l'état qui devient $\mid \psi>=\left[\left|0>+\exp \left(i \phi+i \Delta_{1} \tau_{1}+\mathrm{i} \Delta_{2} \tau_{2}+\ldots\right)\right| 1>\right] / \sqrt{ } 2$. En ajoutant ainsi des sauts de phase aléatoires, on peut perdre complètement trace de la phase initiale $\phi$. Cela sera interprété comme de la décohérence par l'observateur. On voit aussi que la décohérence induit une échelle de temps caractéristique pour la mesure, car toute observation plus rapide que les temps $\tau_{j} n^{\prime} y$ sera pas sensible.

Il faut bien distinguer la notion de déphasage de la décohérence. On parle de déphasage lorsque chaque ion a une fréquence de transition dépendant de son environnement local, mais immuable dans le temps (élargissement inhomogène statique). Si l'on fait une moyenne d'ensemble sur cet élargissement, la phase n'est certes pas définie ; mais par la technique d'écho, on peut remettre en phase les différentes oscillations. Cela veut dire qu'on n'a pas réellement perdu trace de la phase initiale, et ce n'est donc pas de la décohérence au sens propre.

La mesure de cohérence est par excellence l'expérience des oscillations de Rabi (voir p. 49). La continuité des oscillations est assurée par la cohérence entre la vibration atomique et la source excitatrice (fig. E1). Il ne faut pas confondre la fréquence des oscillations de Rabi, qui dépend uniquement de la puissance de la source lumineuse, et la fréquence de transition qu'on appelle fréquence de Larmor en RMN, et qui est beaucoup plus grande (de 10 à $400 \mathrm{MHz}$ selon les isotopes pour un champ magnétique appliqué de 10 teslas).

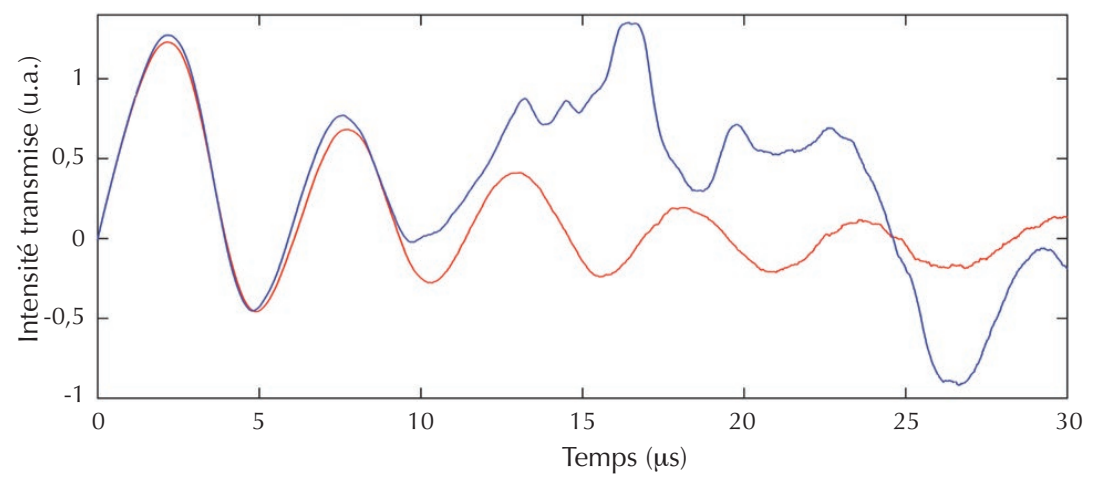

E1. Quand les oscillations de Rabi «tournent mal » (en bleu). Le laser n'est ici pas asservi en fréquence, contrairement à la situation en rouge. Sans asservissement, la « gigue du laser » rend les oscillations chaotiques en fonction du temps. On voit que la cohérence est fondamentalement une notion relative, car ici, c'est le laser qui subit des fluctuations de fréquence. II n'est plus une référence stable pour interroger les atomes et doit donc rester constamment asservi pendant l'expérience.

\section{\>}

\section{Quel support optique d'information?}

Voici une question naïve, mais très fondamentale : pourquoi ne pas utiliser un disque Blu-ray, version moderne du Compact Disc, comme support optique d'information quantique ? Ce n'est en réalité pas possible car, sous le laser de lecture, il y a un grand nombre de molécules photosensibles qui sont ellesmêmes des assemblages complexes d'atomes. Cette complexité est source de décohérence (voir encadré 1), car chaque degré de liberté est une voie possible de perturbation par l'environnement extérieur [2]. Autrement dit, les qubits ne restent pas des superpositions cohérentes une fois inscrits sur le support. Ils se transforment irrémédiablement en états classiques 0 ou 1 .

Pour la réalisation de mémoires quantiques, il est préférable de se tourner vers des systèmes micro ou nanoscopiques simples en faible interaction avec l'environnement extérieur. Les vapeurs atomiques ont été utilisées avec succès pour le stockage d'information quantique [3]. Les atomes du gaz sont en effet bien isolés et peuvent être contrôlés par les techniques modernes de refroidissement laser [4]. L'utilisation de systèmes solides représente un enjeu technologique ambitieux pour la capacité de stockage et leur possibilité d'intégration, mais semble a priori délicate. Par définition, les interactions entre atomes y sont importantes à cause des liaisons chimiques ou ioniques. Les phonons qui traduisent ce couplage intense sont une porte d'entrée pour la décohérence.

Les ions de terres rares sont clairement une exception de ce point de vue.

\section{Cohérence optique des cristaux dopés aux terres rares}

Les cristaux dopés aux terres rares (lanthanides dans le cas présent) sont connus pour leurs propriétés de fluorescence (voir encadré 2). La fluorescence rouge de l'europium était, par exemple, une composante des tubes cathodiques trichromatiques pour la télévision couleur. Dans un autre registre, les cristaux de $\mathrm{Y}_{3} \mathrm{Al}_{5} \mathrm{O}_{12}$ (abrégé YAG) dopés au néodyme sont au cœur de lasers qui portent leur nom : Nd:YAG [5]. Ils servent aussi bien de modestes pointeurs 
lasers verts que de sources de puissance pour l'industrie. La longue durée de vie des niveaux excités associés à cette fluorescence semble un bon point de départ pour la réalisation de mémoires optiques. Elle traduit la capacité à emmagasiner de l'énergie sans que cette dernière s'évanouisse immédiatement dans l'environnement, et permet l'inversion de population nécessaire à l'effet laser (émission stimulée). Cette grande durée de vie n'est pourtant pas suffisante lorsque l'on souhaite stocker un qubit en préservant par définition une superposition cohérente (voir encadré 1).

Comment peut-on vérifier la durée de vie de cette cohérence ? Une mesure cohérente impose de créer une superposition entre deux états. La durée de vie de la population peut être mesurée en observant la décroissance de la fluorescence après une excitation brève. Il faut pouvoir suivre la phase de cette excitation en la comparant, par exemple, au laser incident qui sert d'oscillateur de référence.

\section{Oscillations de Rabi}

L'expérience de référence pour mesurer une cohérence atomique est celle des oscillations de Rabi. Elle requiert une source électromagnétique parfaitement stable. La stabilisation laser, lorsque la transition est dans le domaine optique, est une technique délicate, mais bien maitrisée actuellement [4]. Pour des transitions de plus basse énergie (résonance magnétique nucléaire, RMN), des sources radiofréquence commerciales sont très bien adaptées. C'est dans le cadre de la RMN qu'Isidor Rabi avait développé son modèle et réalisé des expériences à la fin des années 1930. Un système à deux niveaux non dégénérés dont on souhaite tester la cohérence subit une série d'oscillations de population lorsqu'il est soumis à un rayonnement de fréquence adaptée. L'atome passe alternativement de son état fondamental à l'état excité. On peut voir cet échange constant entre atome et champ comme une succession de cycles d'absorption et d'émission stimulée. La fréquence d'oscillation dépend uniquement de la puissance de la source. Les oscillations cessent si on perd la cohérence entre le laser et la vibration élémentaire que représente l'excitation de la transition atomique. L'interaction des atomes avec leur environnement est une source

\section{Cristaux dopés aux ions de terres rares} encadré 2

Les lanthanides et les actinides forment la famille des terres rares. On utilise essentiellement les lanthanides qui ne sont pas radioactifs, contrairement aux actinides. Ils ont des propriétés optiques originales à cause de la structure particulière de la couche électronique incomplète 4f. En effet, les électrons $4 \mathrm{f}$ orbitent plus près du cœur que ceux des couches complètes $5 \mathrm{~s}$ et $5 \mathrm{p}$ (fig. E2a). Par exemple, pour I'ion thulium trivalent, dont la structure $s^{\prime}$ écrit $[\mathrm{Kr}] 4 \mathrm{~d}^{10} 5 \mathrm{~s}^{2} 5 \mathrm{p}^{6} 4 \mathrm{f}^{12}$, les douze électrons $4 \mathrm{f}$ sont en pratique bien protégés de l'environnement extérieur par les huit électrons 5s et 5p, même dans un cristal ionique. Cet isolement donne aux transitions $4 \mathrm{f}-4 \mathrm{f}$ (où on modifie le terme électronique tout en conservant la même configuration électronique) une remarquable finesse, souvent de l'ordre du kHz (élargissement homogène). Cela se traduit théoriquement par des temps de cohérence sur ces transitions d'une fraction de milliseconde à quelques millisecondes à basse température (2K).

En pratique, on n'observe pas des largeurs de raie de l'ordre du kHz car les imperfections cristallines produisent un élargissement inhomogène de l'ordre du GHz, ce qui reste cependant modéré pour des systèmes solides.

Une image de fluorescence d'un cristal dopé avec des ions de terre rare (thulium) est reproduite en figure $\mathrm{E} 2 \mathrm{~b}$.

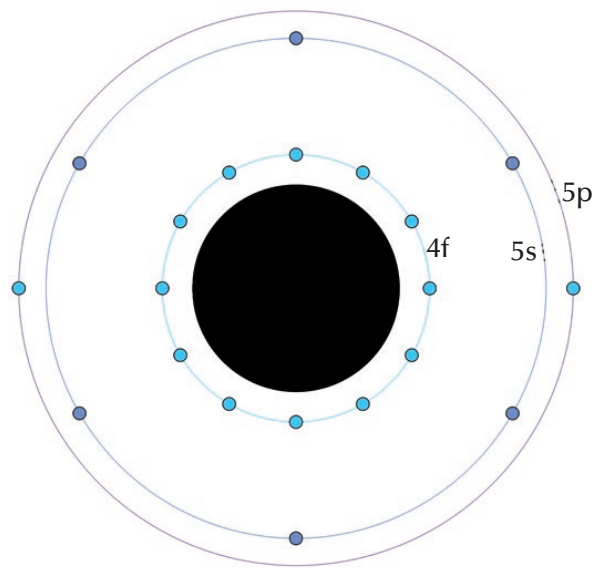

E2a. Schéma simpliste du cortège électronique de l'ion thulium trivalent $\mathrm{Tm}^{3+}$. Les douze électrons $4 \mathrm{f}$ que l'on excite avec le laser (en modifiant le terme électronique, tout en conservant la même configuration électronique) restent bien protégés de l'extérieur par huit électrons $5 s$ et $5 p$, qui forment un écran de protection.

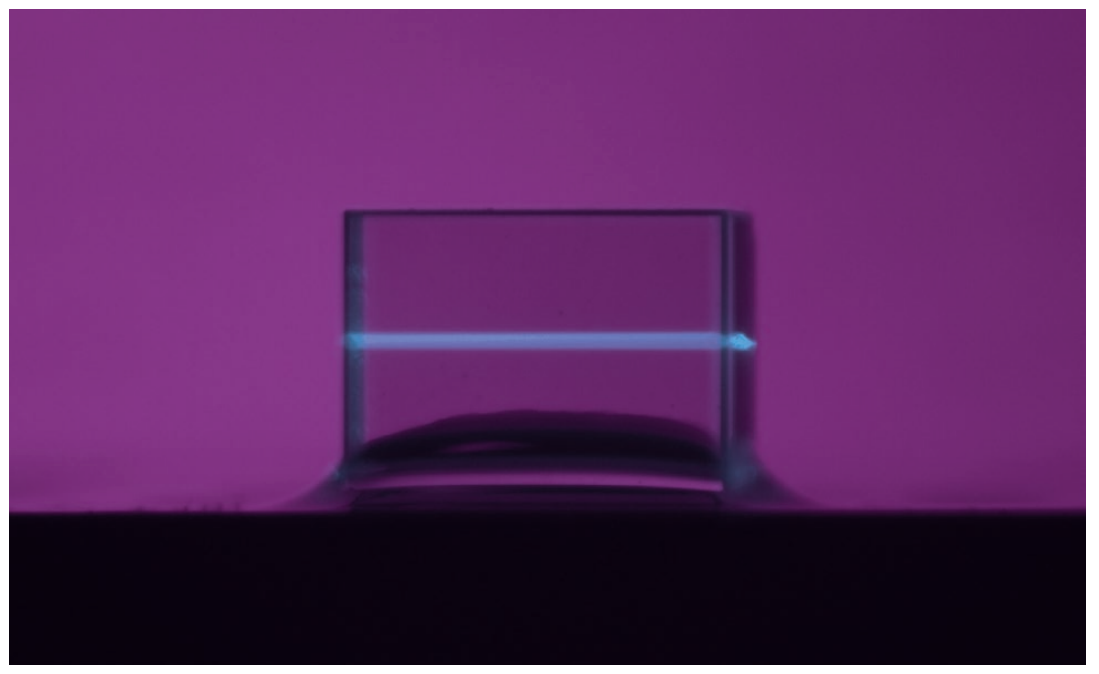

E2b. Fluorescence infrarouge de l'ion $\mathrm{Tm}^{3+}$ dans une matrice de LiNb0 ${ }_{3}$. L'échantillon est en contact thermique sur son support grâce à une colle dont on aperçoit le ménisque. Le trait de fluorescence au centre apparaît flou, car il se superpose à sa réflexion sur la face arrière du cristal (c'est une sorte de « reflet de la physique »!). 
$>>$

de décohérence (voir encadré 1). Les oscillations de Rabi peuvent aussi être dégradées par un bruit technique, sur le laser par exemple. C'est alors la " gigue " en fréquence (ou jitter en anglais) du laser qui produit une décohérence apparente (fig. E1, p. 48). Il ne faut pas confondre la fréquence des oscillations de Rabi, que l'on appelle simplement fréquence de Rabi, et celle de la transition, beaucoup plus grande, que l'on appelle fréquence de Larmor en RMN.

La proposition de Rabi peut être déclinée selon plusieurs variantes, mais reste une brique de base pour les mesures de cohérence. On envoie sur le cristal dopé aux terres rares une impulsion laser dont on enregistre la transmission. Les oscillations de Rabi sont détectées par une baisse ou une augmentation de l'intensité transmise dans le temps. On parle alors de nutation optique (fig. 1). On observe une atténuation des oscillations avec le temps, qui ne peut être attribuée ni à la décohérence par l'environnement ni au bruit technique du laser. Elle provient d'une propriété intrinsèque des cristaux dopés : l'élargissement inhomogène. L'impulsion initiale « arrose » des groupes d'ions dont l'environnement local est différent, ce qui induit un élargissement dit inhomogène de la transition, car à chaque groupe est associée une fréquence différente. On peut montrer que la population de chaque groupe, identifié par son écart de fréquence au laser incident (nommé désaccord, $\delta$ ), oscille avec une fréquence de Rabi qui dépend aussi de $\delta$. C'est la somme de ces différentes oscillations déphasées par le terme en $\delta$ qui atténue la réponse de l'ensemble. On parle plutôt de déphasage, pour ne pas le confondre avec la décohérence qui agit pareillement sur tous les ions. En pratique, on peut compenser ce déphasage par deux techniques similaires : l'écho de spin en RMN et l'écho de photon dans le domaine optique. Cette dernière option nous intéresse plus particulièrement dans le cadre des mémoires quantiques pour la lumière.

\section{L'écho de photon}

L'écho de photon permet de mesurer le temps de vie de la cohérence en s'affranchissant du déphasage qu'impose l'élargissement inhomogène. Cette technique s'inspire de l'expérience de
Rabi, mais en évite les limitations. Au lieu d'une seule impulsion longue, on en applique deux courtes bien séparées dans le temps (fig. 2). La première excite brièvement un ensemble d'ions dans l'environnement inhomogène, chacun caractérisé par son désaccord $\delta$. Les ions évoluent ensuite librement et "vibrent " chacun à leur propre fréquence (de Larmor), accumulant aussi un déphasage. La deuxième impulsion sert à compenser ce dernier. Sa durée est ajustée pour que les ions effectuent une demi-oscillation de Rabi, appelée alors impulsion $\pi$ (une impulsion $2 \pi$ représente une oscillation complète). On peut montrer qu'une impulsion $\pi$ renverse la phase de l'oscillation libre qui a suivi la première impulsion. Elle induit en pratique un rephasage, car la phase accumulée avant l'impulsion $\pi$ est soustraite après. Le rephasage produit un écho optique détectable. À cet instant, les ions émettent en phase indépendamment du désaccord $\delta$. On s'affranchit bel et bien de l'élargissement inhomogène et on a accès aux propriétés de cohérence intrinsèque de l'ion. À titre d'exemple, la cohérence optique de l'ion $\mathrm{Er}^{3+}$ dans une matrice $\mathrm{Y}_{2} \mathrm{SiO}_{5}$ est de quelques millisecondes, alors que la nutation optique, très semblable à la figure 1 , s'amortit en quelques microsecondes. L'écho de photon est un outil puissant de caractérisation des cristaux dopés, et représente une source inépuisable d'inspiration pour concevoir des protocoles de mémoires quantiques.

\section{Écho de photon et mémoire quantique}

De la spectroscopie optique aux protocoles de stockage quantique

Lorsque les cristaux dopés ont été envisagés comme support de mémoire quantique, il était naturel de s'inspirer de l'écho de photon. Lorsqu'on regarde la séquence temporelle de la figure 2 , un changement de point de vue s'impose. On peut voir la première impulsion excitatrice comme un signal à enregistrer, un photon unique par exemple, et l'écho comme sa restitution à un instant ultérieur. L'impulsion $\pi$ entre les deux ne sert qu'à déclencher le rephasage ou, suivant notre point de vue, à relire la mémoire. Ce simple constat fut à l'origine d'un foisonnement de protocoles de mémorisation [6]. L'utilisation d'impulsions $\pi$ intenses dans une séquence mêlant un photon unique en entrée et son écho en sortie est rapidement apparue comme un problème fondamental, car elle a aussi un autre effet que nous avons pour le moment négligé. Elle impose une demi-oscillation de Rabi aux ions qui inverse certes la phase de leur "vibration ", mais elle les promeut aussi dans leur état excité. Cette excitation après l'impulsion $\pi$, et donc au moment de l'écho, est dramatique. L'écho est noyé dans la fluorescence émise par le milieu en train de se désexciter [7].

Au laboratoire Aimé Cotton, notre équipe a récemment proposé une séquence de double rephasage, où l'on enchaîne deux séquences d'écho de photon (fig. 3). On applique au total deux impulsions $\pi$ qui forment finalement une oscillation de Rabi complète et assurent le retour des ions dans leur état fondamental, supprimant ainsi la fluorescence. Nous avons mis en œuvre cette technique dans un cristal d' $\mathrm{Y}_{2} \mathrm{SiO}_{5}$ dopé à l'erbium.

L'utilisation de l'ion $\mathrm{Er}^{3+}$ est un enjeu majeur, car sa longueur d'onde d'excitation est directement compatible avec la bande conventionnelle des télécommunications. Les amplificateurs dopés à l'erbium sont déjà largement présents dans les réseaux fibrés actuels de transport d'information classique. L'ion $\mathrm{Er}^{3+}$ est donc un candidat idéal pour porter aussi l'information quantique.

\section{Réalisation expérimentale dans $\mathrm{Er}^{3+}: \mathrm{Y}_{2} \mathrm{SiO}_{5}$}

Nous avons réalisé une séquence de double rephasage avec $\mathrm{Er}^{3+}: \mathrm{Y}_{2} \mathrm{SiO}_{5}$ (fig. 3). Le signal incident est restitué après l'application de deux impulsions $\pi$. On notera qu'il n'y a pas d'écho entre ces deux impulsions. C'est surprenant a priori car la première impulsion $\pi$ devrait générer un rephasage (écho de photon simple). La seconde assure en fait un rephasage de l'écho simple pour produire un écho double. Il n'est pas concevable pour une mémoire quantique de réémettre deux fois le signal incident sous forme de deux échos consécutifs (simple et double) : on violerait alors un principe fondamental en information quantique appelé le théorème de nonclonage, stipulant que le signal ne peut 


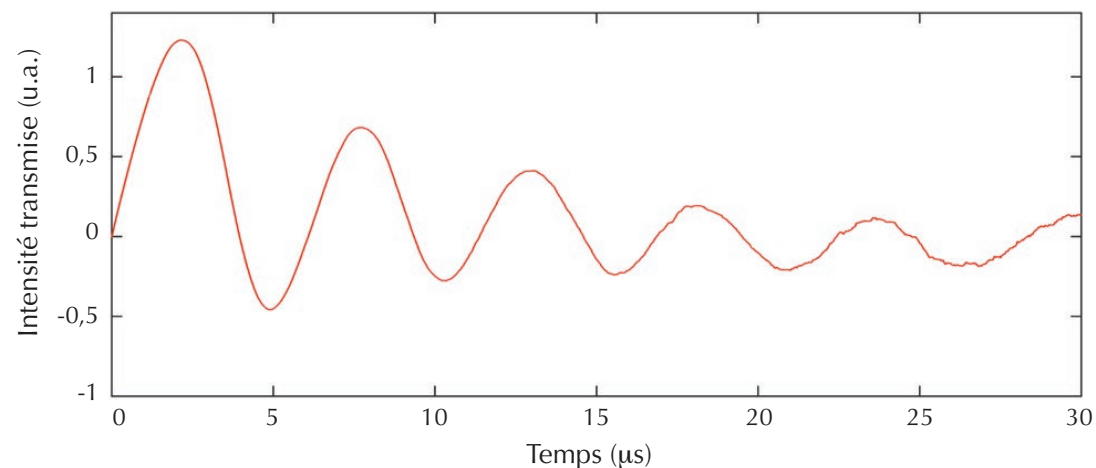

1. Oscillations de Rabi ou nutation optique de l'ion $\operatorname{Tm}^{3+}$ dans $\mathrm{Y}_{3} \mathrm{Al}_{5} \mathbf{0}_{12}$. On les observe directement sur l'évolution temporelle de la transmission d'un faisceau intense. L'atténuation des oscillations est due à la présence d'un élargissement inhomogène (voir encadré 1 et p. 50).

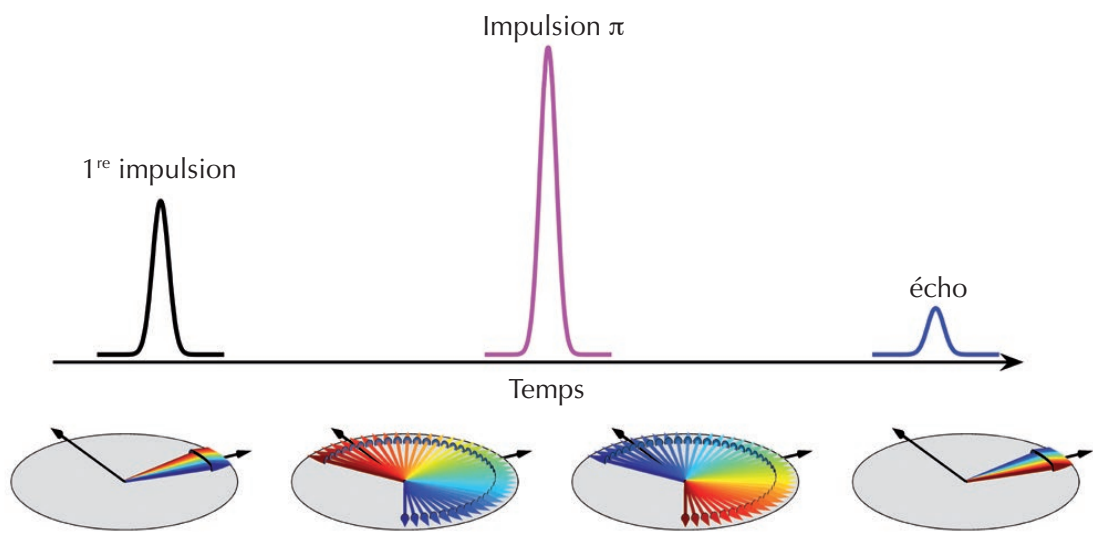

2. La technique d'écho de photon. (Haut) Séquence temporelle. Une première impulsion excite brièvement les ions. L'impulsion $\pi$ renverse la phase de l'oscillation pour produire un écho. (Bas) Représentation graphique du déphasage et du rephasage produits par l'élargissement inhomogène. Pour chaque désaccord $\delta$, on représente une flèche qui tourne sur le cercle unité à la vitesse angulaire $\delta$ décrivant la trajectoire complexe

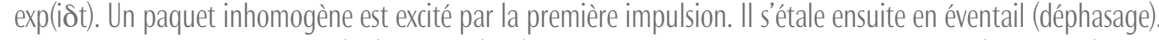
L'impulsion $\pi$ transforme $\exp (i \delta t) \rightarrow \exp (-\mathrm{i} \delta t)$. L'éventail une fois renversé se referme (rephasage) pour produire l'écho. Cette image est largement utilisée en RMN pour décrire le mouvement des spins nucléaires.

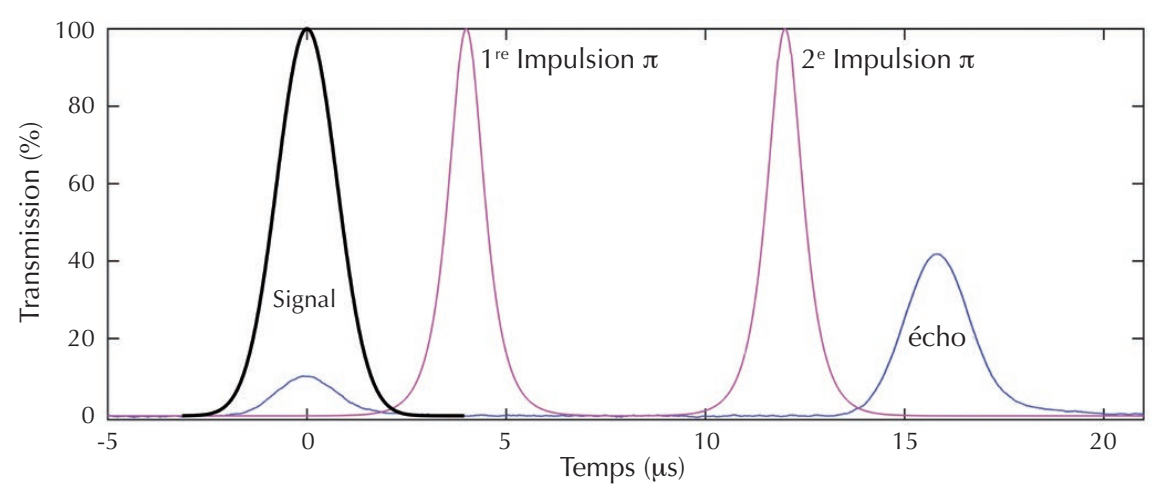

3. Séquence expérimentale de double rephasage avec $\mathrm{Er}^{3+}: \mathrm{Y}_{2} \mathrm{Si}_{5}$. La première impulsion est le signal à mémoriser (en noir). II est absorbé à hauteur de 90\%, laissant seulement échapper 10\% du signal transmis (en bleu). Après deux impulsions $\pi$, on retrouve $40 \%$ du signal sous forme d'écho. être relu qu'une fois et obligatoirement lorsque les ions sont dans l'état fondamental. On voit qu'effectivement sur la séquence de la figure 3, l'écho n'est émis qu'à la fin, après les deux impulsions $\pi$. L'écho simple, malgré le rephasage, est rendu "muet " dans notre cas par le protocole que nous avons prénommé ROSE pour "Revival Of Silence Echo", dont une explication détaillée sort du cadre de cet article.

L'efficacité de stockage est de $40 \%$ si l'on compare l'écho à l'impulsion incidente [8]. C'est encourageant. Le temps de stockage est ici de $16 \mu$ s pour une démonstration de principe, limité cependant par le temps de cohérence du matériau, de l'ordre de $4 \mathrm{~ms}$. Cette dernière durée peut paraître modeste, mais elle correspond déjà au temps de propagation dans une fibre entre Lille et Nice $(1200 \mathrm{~km})$ !

\section{Conclusion}

Beaucoup reste à faire avant de considérer les mémoires quantiques comme des briques fiables, efficaces et rapides, d'une version quantique de notre internet. Leur développement permet avant tout une compréhension des matériaux à leur échelle la plus fondamentale, en posant la question de la réalisation pratique d'un assemblage de systèmes microscopiques pour former finalement un objet macroscopique régi par la mécanique quantique. Ces avancées conceptuelles feront sans doute germer derrière elles des applications que nous n'envisageons pas encore.

\section{Références}

1• A. Beveratos, « Cryptographie quantique en sécurisation des réseaux. Situation et perspectives", Techniques de l'ingénieur, base documentaire : TIB454DU0, nm2400 (2008).

2- M. Le Bellac, Reflets de la physique 13 (2009) 15-18

3• T. Chanelière et J. Laurat, Photoniques 71 (2014) 29-33.

4 - M. Leduc et P. Lemonde, Reflets de la physique 21 (2010) 46-51.

5• S. Forget, I. Robert-Philip et P. Balcou, Reflets de la physique 21 (2010) 18-23.

6・ W. Tittel et al., Laser Photonics Reviews, 4(2) (2010) 244-267.

7• C. Schwob et L. Julien, Reflets de la physique 21 (2010) 12-16.

8• J. Dajczgewand et al., Optics Letters, 39 (2014) 2711. 